\title{
Semi-Analytic Theory of Similariton Amplifiers and Laser Oscillators Using a Shape-Adaptive Model Pulse
}

\author{
Christian Jirauschek $^{1 *}$ and F. Ömer Ilday ${ }^{2}$ \\ ${ }^{1}$ Institute for Nanoelectronics, Technische Universität München, Arcisstraße 21, D-80333 Munich, Germany \\ ${ }^{2}$ Department of Physics, Bilkent University, 06800, Ankara, Turkey \\ *jirauschek@mytum.de
}

\begin{abstract}
A semi-analytic theory for similariton lasers and amplifiers is presented. The key is a shape-adaptive model pulse which can be continuously tuned to represent pulse shapes ranging from parabolic to Gaussian to sech-squared intensity profiles.

(C) 2011 Optical Society of America

OCIS codes: $060.5530,060.7140$.
\end{abstract}

\section{Introduction}

In recent years, self-similar propagation of short optical pulses was reported in fiber amplifiers as well as laser resonators $[1,2]$. For self-similar evolution, the pulse approaches a parabolic intensity profile. As the Kerr nonlinearity just induces a phase shift proportional to the pulse profile, its effect is reduced to merely adding a quadratic phase, corresponding to a linear chirp. Thus, higher pulse energies can be obtained since wave breaking is avoided. Similariton lasers are now considered as constituting a new mode-locking regime, distinct from the solitary, stretched-pulse and all-normal-dispersion regime. From a practical standpoint, these lasers have led to significantly increased pulse energies. Recently, the soliton-similariton laser has been reported as a further type of mode-locked laser, where alternate propagation in the self-similar and soliton regimes occurs [3].

From both a fundamental and a technical point of view, there is a strong need to improve the physical understanding of fiber amplifiers and lasers working in the self-similar regime. In this context, theoretical modeling is essential. While full numerical simulations yield good agreement with experiment [1-3], they are computationally demanding. On the other hand, compact analytical or semi-analytic models are valuable for an improved intuitive understanding of the self-similar pulse evolution in such systems, furthermore allowing for design optimization over a vast parameter range. Exact analytical self-similar solutions are only asymptotically reached [1]. Thus, semi-analytic approaches have been employed, yielding evolution equations for the characteristic pulse parameters, such as the pulse energy and duration. However, such approaches typically rely on fixed pulse shapes such as Gaussian or sech pulses [4-6], apart from very few exceptions [7].

Here, we present a semi-analytic theory for the pulse propagation in strongly nonlinear optical systems, such as similariton lasers and amplifiers. The key is a shape-adaptive model pulse which can be continuously tuned by a single parameter to represent pulse shapes ranging from parabolic to Gaussian to sech-squared (i.e., soliton-like) intensity profiles. This allows us to adequately describe the pulse propagation in different nonlinear regimes, thus constituting a simple unified theoretical approach.

\section{Shape-Adaptive Model Pulse}

The optical pulse propagation through a dispersive fiber with Kerr nonlinearity can be described by a generalized nonlinear Schrödinger equation. The Gaussian pulse is an exact solution in the linear case and a good approximation for weak nonlinearity. On the other hand, for self-similar propagation in the strongly nonlinear limit, the pulse approaches a parabolic intensity profile. In the anomalous dispersion regime, sech-shaped soliton solutions exist (i.e., featuring a sech-squared intensity profile). For the propagation through a fiber segment, the pulse shape evolves continuously, e.g., approaching a parabolic shape in a similariton amplifier. In fiber lasers, different propagation regimes alternate, leading to a pronounced periodic change of the pulse shape. For example, in the soliton-similariton laser, self-similar pulse evolution occurs in the gain fiber, followed by Gaussian filtering and subsequent soliton-like propagation in the 
anomalous dispersion segment. Thus for a semi-analytic description of strongly nonlinear systems, a versatile model pulse is required, capable of continuously adapting to the various pulse shapes.

We report a model pulse which can be continuously tuned between a parabolic and a Gaussian shape, and can to a very good approximation also describe sech-squared intensity profiles. The starting point is a function $p_{n}(\tau)=$ $\exp \left(-\sum_{k=1}^{n} \tau^{2 k} / k\right)$, which represents a Gaussian for $n=1$, and approaches a parabolic profile for $n \rightarrow \infty$, with $p_{n}=$ $1-\tau^{2}$ for $\tau^{2} \leq 1$ and $p_{n}=0$ for $\tau^{2}>1$ [8]. Since $n$ is not a continuous variable, the pulse shape can only be adapted in discrete steps. This limitation can be overcome by rewriting $p_{n}$ in closed form,

$$
p_{n}(\tau)=\left(1-\tau^{2}\right) \exp \left\{n^{-1}|\tau|^{2 n}\left[{ }_{2} \mathrm{~F}_{1}\left(1, n ; 1+n ; \tau^{2}\right)-1\right]\right\}
$$

Here, ${ }_{2} \mathrm{~F}_{1}$ is the Gauss hypergeometric function, which is routinely implemented in many mathematical tools. The tuning parameter $n$ can now assume arbitrary positive values, offering much more versatility for representing different pulse shapes. Specifically, for $n \approx 0.5, p_{n}$ describes to a very good approximation a sech-squared intensity profile, corresponding to the shape of a fundamental optical soliton. For the pulse envelope $u(z, t)$, we formulate an ansatz $u=A p_{n}^{1 / 2} \exp \left(\mathrm{i} \beta \tau^{2}+\mathrm{i} \alpha \tau^{4}+\mathrm{i} \phi\right)$, where $z$ and $t$ denote the position and retarded time, and $\tau=t / T$ is the normalized time. Furthermore, $A, T, \beta, \alpha$, and $\phi$ denote the amplitude, pulse duration, linear and third order chirp parameter, and phase, respectively. Applying the method of moments [4] to the governing propagation equation, evolution equations can be formulated for the pulse parameters, which are taken to be functions of the position $z$. Specifically, an evolution equation is set up for $n(z)$, allowing to monitor the change of the pulse shape during propagation.

\section{Results}

In Fig. 1, the semi-analytic solution based on our shape-adaptive model pulse is compared to exact analytical and numerical solutions for various cases. In Fig. 1(a), the fundamental soliton solution in the anomalous dispersion regime is shown. Our model pulse excellently reproduces the $\operatorname{sech}^{2}$ intensity profile of the exact solution. In Fig. 1(b) and (c), the intensity profile and power spectrum are shown for a Gaussian initial pulse with 0.2 ps duration (full width at halfmaximum) after $3 \mathrm{~m}$ propagation in a similariton amplifier [1]. The model pulse captures very well the pulse evolution towards a similariton, reproducing the nearly parabolic profile and also the spectral pulse features.
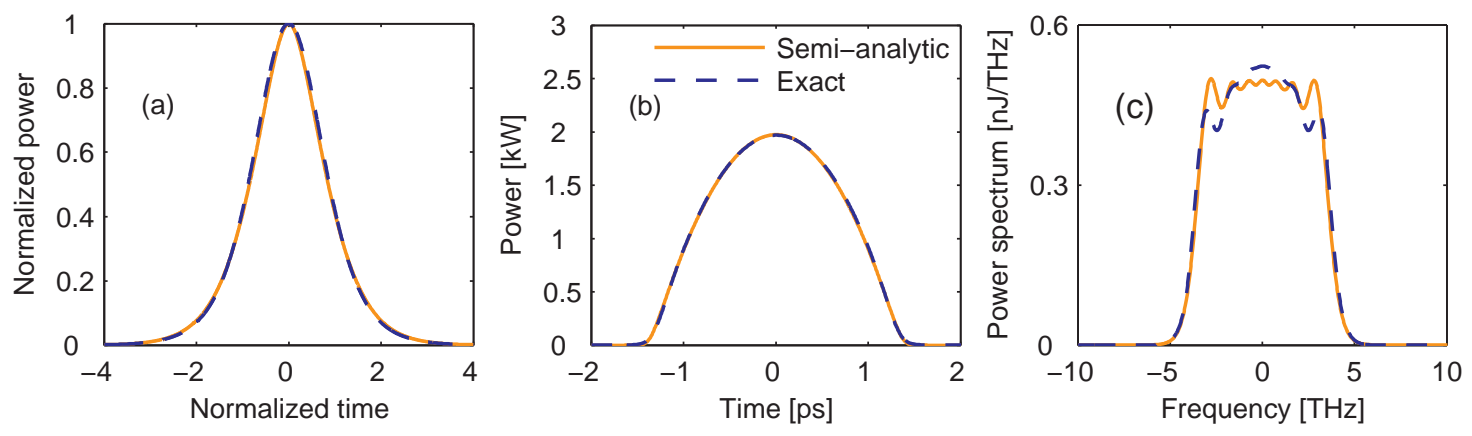

Fig. 1. Comparison of semi-analytic and exact pulse. (a) Fundamental soliton solution for medium with anomalous dispersion. (b) Intensity profile and (c) power spectrum of a pulse after propagation in a similariton amplifier.

In Fig. 2, simulation results are shown for a soliton-similariton laser [3], consisting of a gain fiber, a section of single mode fiber (SMF) with anomalous dispersion, a saturable absorber (SA), spectral filter, and again a section of SMF. The semi-analytic and exact numerical steady state solutions are shown at different positions in the cavity, as indicated in Fig. 2. The model pulse excellently reproduces the nearly parabolic intensity profile after the gain fiber (Fig. 2(a)) and the profile at the entrance of the SA (Fig. 2(b)), as well as the sech ${ }^{2}$-like shape after propagation through the anomalous dispersion fiber (Fig. 2(c)). In Fig. 2(d), the pulse shape evolution in the resonator is visualized, characterized by the parameter $s=n /(n+1)$; i.e., $s=0.5$ corresponds to a Gaussian, $s=1$ to a parabolic and $s \approx 0.33$ to a $\operatorname{sech}^{2}$-like intensity profile. For the numerical result, $s$ is determined by calculating the kurtosis (i.e., fourth standardized moment) of the obtained intensity profile and determining the value of $n$ for our model pulse Eq. (1) 
which yields the same kurtosis. The pulse shape evolution is very well reproduced by our semi-analytic approach in the different nonlinear propagation regimes of the soliton-similariton laser.

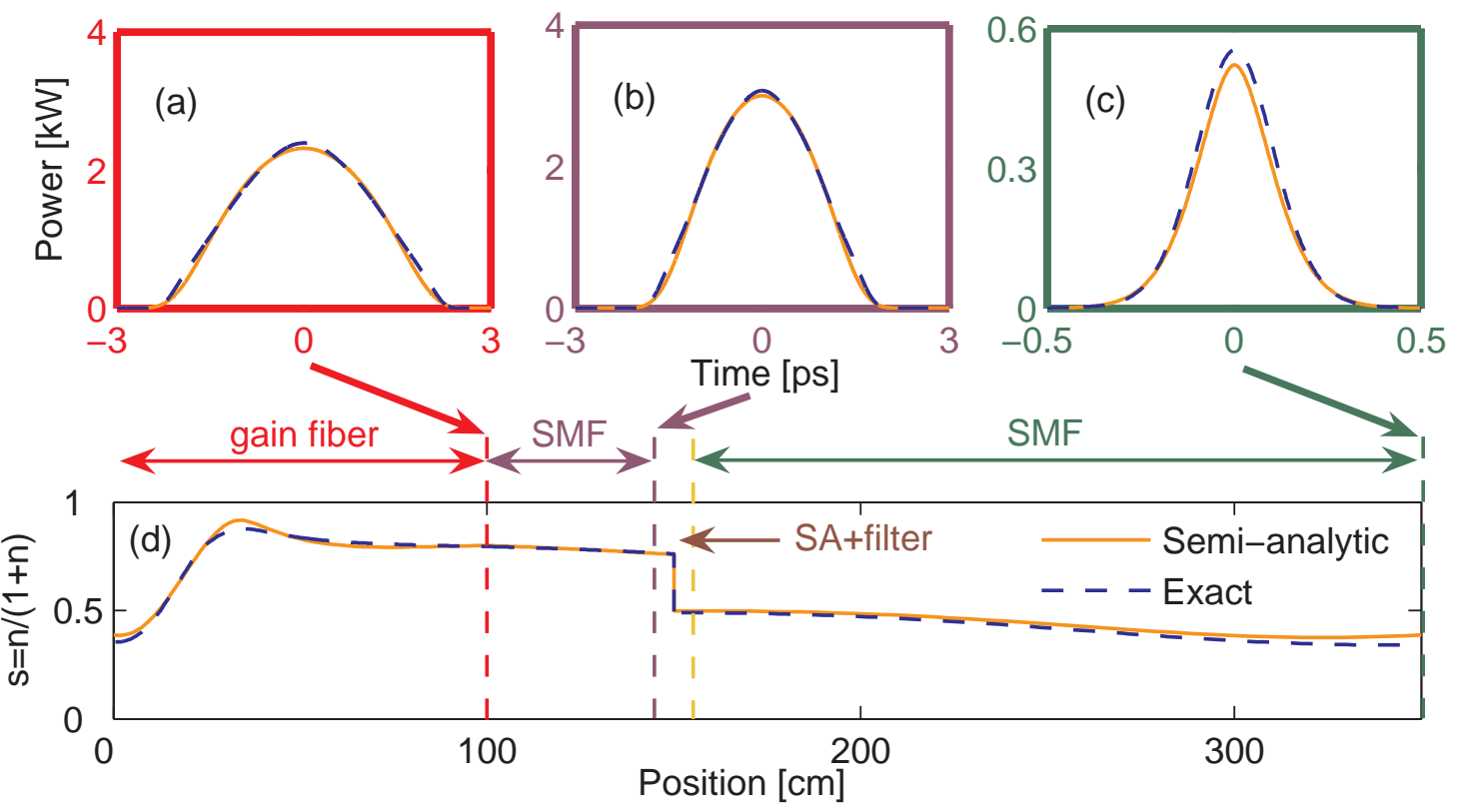

Fig. 2. (a)-(c) Semi-analytic and exact intensity profile at different positions in the soliton-similariton laser, and (d) evolution of the pulse shape parameter $s$ along the cavity.

These results demonstrate that our shape-adaptive model pulse works very well for quite different regimes of nonlinear optical propagation. Furthermore, our approach constitutes the first semi-analytic model for the soliton-similariton laser. Due to the versatility of our model pulse, we expect it to prove useful also for other applications in nonlinear optics and beyond.

C.J. acknowledges support from the German Research Foundation (DFG) within the Emmy Noether program (JI 115/1-1) and under DFG Grant No.JI 115/2-1. F.Ö.I. acknowledges support by the Scientific and Technological Research Council of Turkey (TÜBİTAK) Project No.109T350 and Project No.209T058 and by the EU 7th Framework Project CROSS TRAP Grant No.244068.

\section{References}

1. M. E. Fermann et al., "Self-similar propagation and amplification of parabolic pulses in optical fibers," Phys. Rev. Lett. 84, 6010-6013 (2000).

2. F. Ö. Ilday, J. R. Buckley, W. G. Clark, and F. W. Wise, "Self-similar evolution of parabolic pulses in a laser," Phys. Rev. Lett. 92, 213902 (2004).

3. B. Oktem, C. Ülgüdür, and F. Ö. Ilday, ”Soliton-similariton fibre laser,” Nat. Photonics 4, 307-311 (2010).

4. E. N. Tsoy, A. Ankiewicz, and N. Akhmediev, "Dynamical models for dissipative localized waves of the complex Ginzburg-Landau equation," Phys. Rev. E 73, 036621 (2006).

5. C. Jirauschek and F. X. Kärtner, "Gaussian pulse dynamics in gain media with Kerr nonlinearity," J. Opt. Soc. Am. B 23, 1776-1784 (2006).

6. B. G. Bale, S. Boscolo, J. N. Kutz, and S. K. Turitsyn, "Intracavity dynamics in high-power mode-locked fiber lasers," Phys. Rev. A 81, 033828 (2010).

7. C.-J. Rosenberg et al., "Evolution of optical pulses towards wave breaking in highly nonlinear fibres," Opt. Commun. 273, 272-277 (2007).

8. C. Jirauschek, F. Ö. Ilday, and F. X. Kärtner, ”A Semi-Analytic Theory of the Self-Similar Laser Oscillator,” in Nonlinear Guided Waves and Their Applications (NLGW), Technical Digest (CD), paper WC4 (OSA, 2005). 